\title{
High Isolation Planar UWB Antennas for Wireless Application
}

\author{
Alaa H. Radhi, R. Nilavalan, H. S. Al-Raweshidy and Nur Ab Aziz \\ College of Engineering, Design \& Physical Science, Brunel University, London, UK. \\ Email: Alaa.Radhi@brunel.ac.uk
}

\begin{abstract}
A new printed ultra-wide band (UWB) antenna system is proposed for MIMO applications. The proposed antenna system consists of two identical circular disc monopole elements etched on a single FR4 substrate with a compact size of $93 \times 47 \times 1.6 \mathrm{~mm}^{3}$ and is fed by a $50-\Omega$ microstrip line. The proposed antenna can operates over UWB range from 3.1 to $10.6 \mathrm{GHz}$. A novel compact planar decoupling structure is inserted into the coupled antenna array to increase the isolation; aprecise performance study has been implemented to investigate the mutual coupling reduction effects. Therefore, a good isolation of less than -31dB has been achieved through the entire UWB band (more than $12 \mathrm{~dB}$ improvement over the reference) for an antenna spacing less than $0.3 \lambda$. .The antenna is an excellent candidate in UWB applications for handsets or portable laptops using multiple-input-multiple-output (MIMO) or diversity systems.
\end{abstract}

Keywords-Ultra Wide Band (UWB); mutual coupling reduction; monopoles Antenna; Multiple-Input-Multiple-Output (MIMO).

\section{INTRODUCTION}

Recently, developments in modern wireless communication systems have imposed additional challenges on antenna designers to produce new designs that are miniaturized and have broadband performance [1].With the rapid expansion of wireless MIMO communication systems, low profile, wide bandwidth, high isolation between antennas that maintain good performance is still in high demand. In MIMO systems, multiple antenna elements are required at both receiver and transmitter side [2]. As a result, the design of two or more antennas on a small mobile terminal for the MIMO systems is more challenging compared to the design of a conventional single antenna [3]. Since MIMO systems employ multiple antennas, require high decoupling between antenna elements. However, installing multiple antenna elements in the small space available in portable devices will inevitably cause severe mutual coupling and significantly degrades the diversity performance. Thus one of the main challenges to employing MIMO systems in portable devices is the design of small MIMO antennas with lowest mutual coupling [2]. Efforts have been directed to eliminate or decrease the effects of mutual coupling between different microstrip antennas working in MIMO applications. Among the main mutual coupling reduction techniques that have been extensively studied and discussed in the literature for mutual coupling reduction in UWB-MIMO applications are the following: Neutralization Line (NL) [4], insertion of Electromagnetic Band Gap (EBG) structure [3], spatial and angular variation technique [5-6], inserting a stub structure on ground [7-8], Defected Ground
Structure (DGS) structure [9], implementation of partial ground plane [10].etc. In this paper, UWB-MIMO antennas with a broadband bandwidth ranging from 3.1 to $10.6 \mathrm{GHz}$ have been proposed. Despite the compact size, the inclusion of the proposed structure improves the isolation and effectiveness of the MIMO design.

\section{ANTENNA DESIGN AND CONFIGURATION}

\section{A. Design Procedures of Proposed Antenna}

The initial MIMO antenna configuration is shown in Fig. 1. It consists of two symmetrical circular disc-shaped monopole radiating elements denoted as Ant-1- and Ant-2-, which are located on the same sides (Left and Right) of the FR4 substrate with a permittivity $\varepsilon_{r}=4.4$ (loss tangent=0.018) with a $50-\Omega$ microstrip feed line (as shown in Fig. 1). The antenna is formed by a metallic disc of radius $(R)$, and it has a partial ground plane with a length $(L g)$, and a width $(W g)$ as shown in Fig. 1. The antenna is analysed and optimised by Ansoft HFSS software ver 17.0 achieving the goal of reflection coefficient less than $-10 \mathrm{~dB}$. The dimensions obtained after optimization are as the following: substrate length $L s=93 \mathrm{~mm}$, substrate width $W s=47 \mathrm{~mm}$, substrate height $\mathrm{h}=1.6 \mathrm{~mm}$, gap $g=0.5 \mathrm{~mm}$, ground plane length $L_{g}=47 \mathrm{~mm}$, ground plane width $W_{g}=10.5 \mathrm{~mm}$, feed line length $L_{f}=12.5 \mathrm{~mm}$, feed line width $W_{f}=1.5 \mathrm{~mm}$ and element radius $R=12 \mathrm{~mm}$, space $S=$ $0.3 \lambda_{0}$ (centre to centre separation). two rectangular slots (denoted by $S_{1}$ and $S_{2}$ ) of dimensions $1.6 \mathrm{~mm} \times 6 \mathrm{~mm}$ were introduced on the common ground plane for better impedance matching.

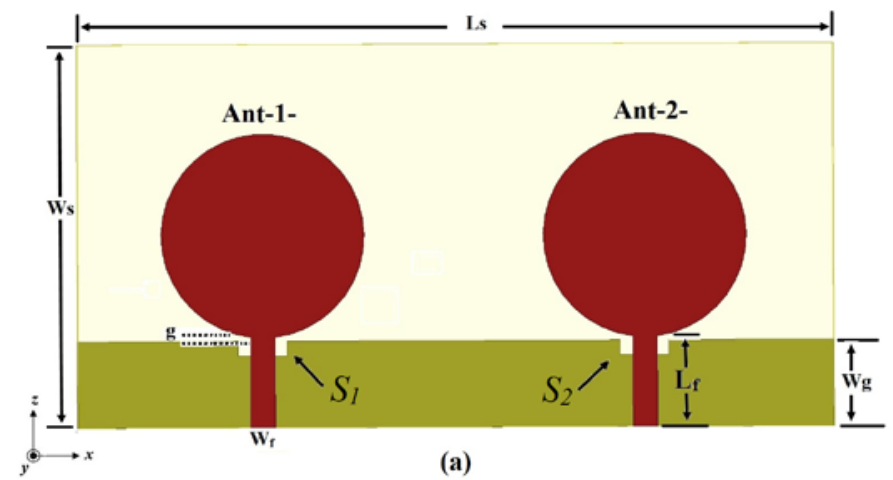

Fig. 1. Layout of the proposed antenna without the planar structure (Top view)

B. The Planar Structure for Mutual Coupling Reduction

A new decoupling structure has been proposed between the antenna elements to improve the ultra-wide band isolation 


\section{Draft Version - Brunel University}

between the two radiating elements. a planar structure has been chosen which composes of modified patch like $\mathrm{T}$ on the upper side (with optimized dimensions $36 \mathrm{~mm} \times 9 \mathrm{~mm}$ ); while a smaller patch like inverse $\mathrm{T}$ on the lower side (with dimensions $17 \mathrm{~mm} \times 6 \mathrm{~mm}$ ) are joint each other and subtracted by four half-circular slots (two half-circular in the upper side while other two smaller half-circular in the lower side as shown in Fig. 2) to form a compact parasitic strip that has been applied as planar decoupling structure between dual-monopoles antennas to have lowest possible mutual coupling instead of other conventional structures which may occupy larger space between the antennas.

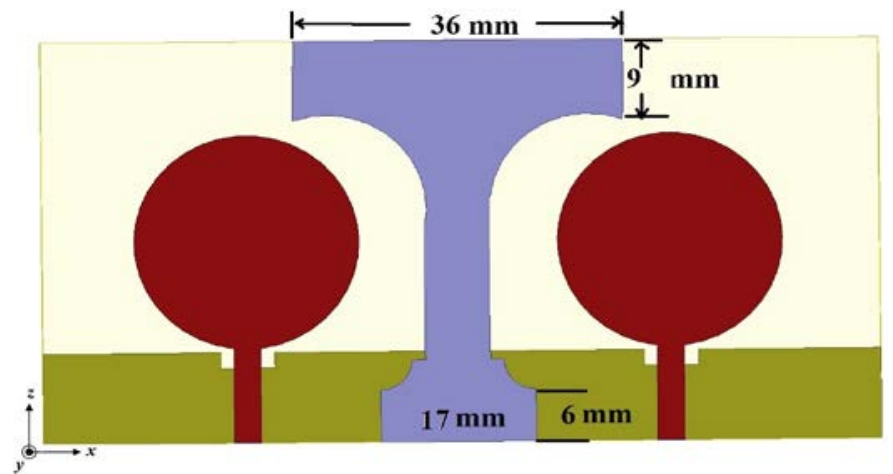

Fig. 2. The layout of the proposed UWB antenna separated by the planar structure. (Top view)

\section{RESULTS AND DISCUSSION}

\section{A. S-Parameters}

The mutual coupling between antenna elements can be determined from $S_{21}$ and $S_{12}$ parameters; By inserting the planar structure between UWB antennas, better isolation of less than $-31 \mathrm{~dB}$ has been achieved through the entire UWB band $(<$ $12 \mathrm{~dB}$ improvement over the reference, for an antenna spacing less than $0.3 \lambda_{0}$ ) as shown in Fig. 3.

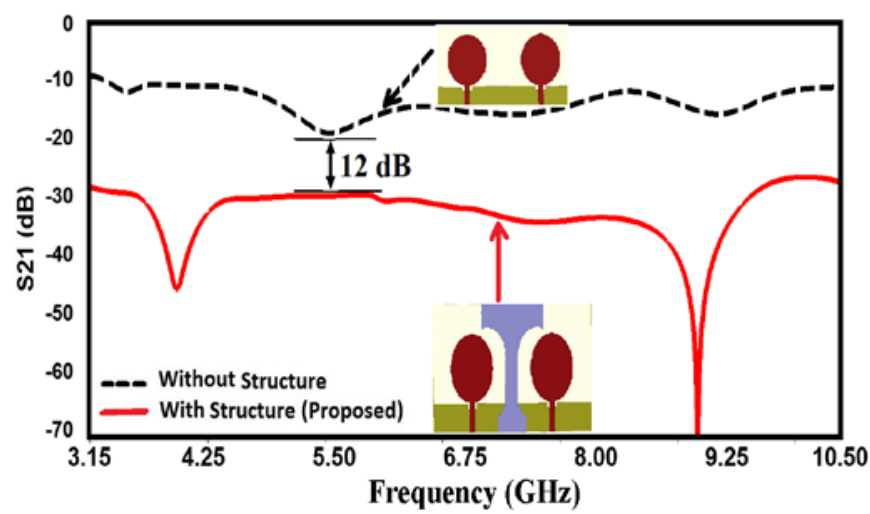

Fig. 3. Simulated $S_{21}$ of the proposed UWB-MIMO antennas.

Fig. 4. illustrates the reflection coefficient performance; it was observed that the antennas in both cases (without and with structure) has a broadband bandwidth ranging from 3.1 to 10.6 $\mathrm{GHz}$ (for $\mathrm{S}_{11}<-10 \mathrm{~dB}$ ). Thus the antenna satisfies the impedance matching requirement for the entire UWB range specified by the FCC.

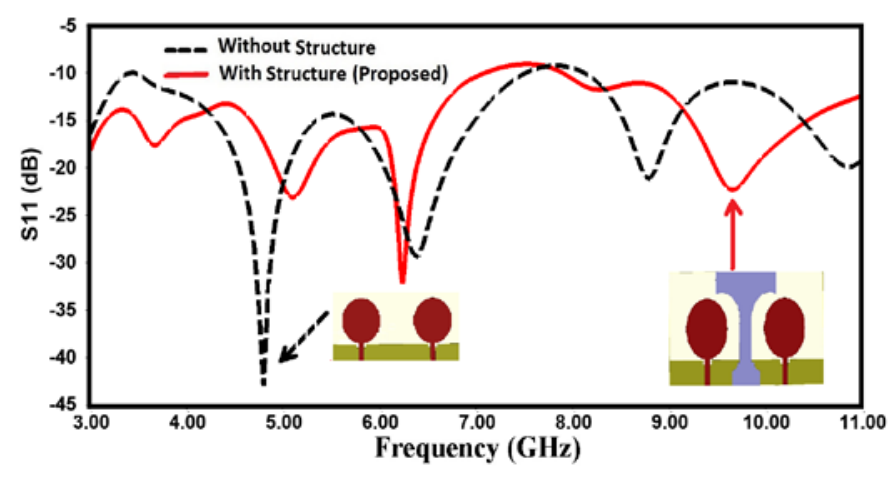

Fig. 4. Simulated $\mathrm{S}_{11}$ of the proposed UWB-MIMO antennas

\section{B. Simulated Radiation Patterns, Gain and Efficiency}

This section presents the study performed on the effect of far-field radiation patterns as a comparison between both cases (with and without inserting the structure). The two orthogonal-plane patterns of the antenna with absence and presence of the structure are demonstrated in Fig. 5 (at the middle frequency of UWB range), in the principal planes at $\theta=$ $90^{\circ}$ and $\varphi=90^{\circ}$ which corresponding to the $x-y$ and $y-z$ planes, respectively.

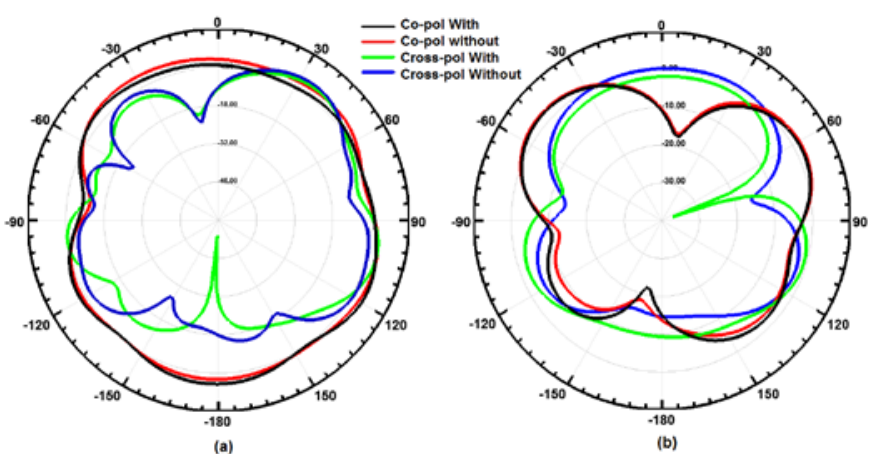

Fig. 5. Simulated Radiation patterns of the two monopoles array with and without structure at $7 \mathrm{GHz}$. (a) x-y plane, (b) y-z plane.

Overall antenna radiation patterns are relatively stable across different UWB frequencies (still nearly omnidirectional). However; the decoupling structure does not affect or disturb antenna patterns significantly. These plots were obtained with one antenna port excited and other port terminated with matched impedance i.e. $50 \Omega$ load.
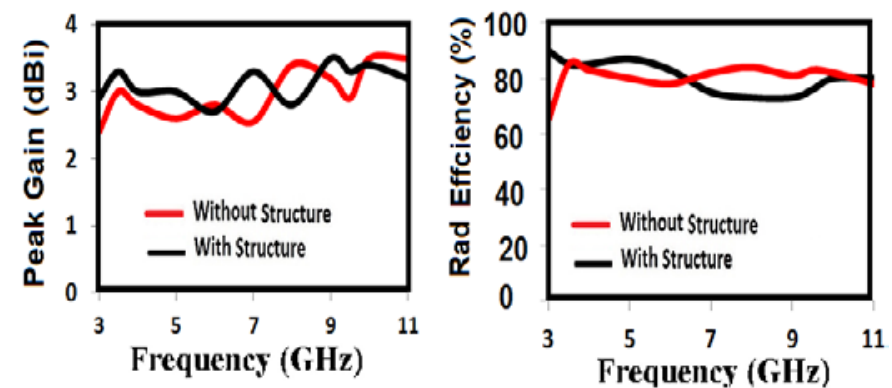

Fig. 6. Simulated peak gains (Left) and radiation efficiencies (Right) of the proposed UWB antenna.

In addition, the simulated peak gain and simulated radiation efficiency at different frequencies are plotted in Fig. 6. The achieved gain for the proposed array without and with structure 
in the UWB frequency band is $2.5-3.3 \mathrm{dBi}(0.8 \mathrm{dBi}$ gain variation) and 2.9-3.2 $\mathrm{dBi}(0.3 \mathrm{dBi}$ gain variation $)$, respectively. After inserting structure; It is also observed that the radiation efficiency of the antenna is still high (about 88\%) in the most of UWB frequency band white it drops in other higher frequencies (as shown in Fig. 6.).

\section{Surface Current Distribution}

The effects of the structure can also be analyzed through surface current distribution and is described in this section as shown in Fig. 7 at middle UWB range. When one port is excited, a significant portion of surface currents is coupled from Ant-1- towards Ant-2- through the common ground plane and the substrate (without structure case). However, the direct coupling current flow between two antenna ports is reduced significantly by inserting the decoupling structure.

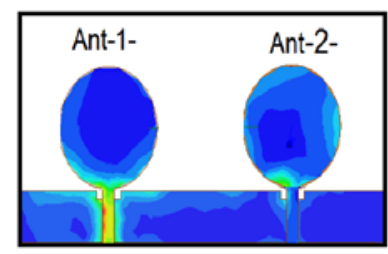

a
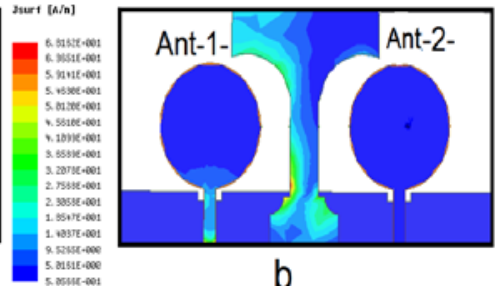

b
Fig. 7. Simulated surface current density at $7 \mathrm{GHz}$ of the antenna array.(a) Without case (b) With case.

It is observed from Fig. 7. a very less amount of current is induced in the second antenna because most of the antenna's direct coupling field are concentrated in the planar structure; this shows the creation of additional current paths between the two radiating antennas and prevents most of the induced currents to be transferred towards the other antenna element. The effect is same when Ant-2- was excited while Ant-1- was terminated.

\section{COMPARISON WITH OTHER APPROACHES}

As mentioned before; other approaches has also been applied to reduce mutual coupling between UWB-MIMO antennas: insertion of EBG structures, defected or slotted ground plane, spatial and angular variations technique, insertion of a resonator in between.etc. The table below shows the comparison between the design presented in this paper and other recent published works working in UWB applications (BW ranging from 3.1 to $10.6 \mathrm{GHz}$ ); the proposed antennas clearly show the highest isolation with reduced physical space between antennas.

TABLE I. PERFORMANCE COMPARISON OF MUTUAL COUPLING REDUCTION FOR UWB ANTENNAS

\begin{tabular}{|c|c|c|c|c|}
\hline Ref & $\begin{array}{c}\text { Min } \\
\text { Isolation } \\
(\mathrm{dB})\end{array}$ & $\begin{array}{c}\text { Volume } \\
\left(\mathrm{mm}^{3}\right)\end{array}$ & $\begin{array}{c}\text { Space } \\
\left(\lambda_{0}\right)\end{array}$ & Technique \\
\hline$[4]$ & -20 & $100 \times 50 \times 8$ & 0.4 & Inserting a EBG \\
\hline$[6]$ & -20 & $60 \times 60 \times 1.6$ & 0.4 & Spatial variation \\
\hline$[8]$ & -18 & $68 \times 40 \times 1.6$ & 0.3 & Inserting a stub \\
\hline$[9]$ & -19 & $48 \times 25 \times 1.6$ & 0.5 & DGS structure \\
\hline
\end{tabular}

\begin{tabular}{|c|c|c|l|l|}
\hline$[10]$ & -17 & $91 \times 38 \times 1.6$ & 0.4 & Partial ground plane \\
\hline Proposed & -31 & $93 \times 47 \times 1.6$ & 0.3 & $\begin{array}{l}\text { planar structure in } \\
\text { between }\end{array}$ \\
\hline
\end{tabular}

\section{CONCLUSIONS}

In this paper, a new compact MIMO antenna with high isolation has been proposed for UWB applications. A novel planar structure has been proposed for mutual coupling reduction between two identical circular disc monopole antennas for UWB-MIMO applications. The bandwidth of the proposed antenna covers almost the entire UWB ranging from 3.1 to $10.6 \mathrm{GHz}$. The planar decoupling structure is shown to be useful in achieving compactness and better isolation (less than $-31 \mathrm{~dB}$ is obtained through the whole UWB frequency range). A precise performance study, involving impedance B.W, isolation, radiation pattern, and surface current density distribution, was performed to identify the significance of the planar structure. The obtained results of the bandwidth characteristics and isolation show that the proposed antennas is suitable for MIMO applications and can work well in the UWB applications for some portable devices. As future work, practical implementation of the design and simultaneous optimisation using standard optimisation procedures will be considered.

\section{REFERENCES}

[1] K. M. Prasanna and S. K. Behera, "Compact two-port UWB MIMO antenna system with high isolation using a fork-shaped structure,” 2013 International Conference on Communication and Signal Processing, 2013.

[2] C. A. Balanis, Antenna Theory: Analysis and Design, 3rd Edition. Hoboken, NJ: John Wiley \& Sons, Inc., 2005.

[3] Z. Z. Abidin, R. A. Abd-Al Hameed, N. J. Mcewan, and M. B. Child, "Analysis of the effect of EBG on the mutual coupling for a twoPIFA assembly,” 2010 Loughborough Antennas \& Propagation Conference, 2010.

[4] S. Zhang and G. F. Pedersen, "Mutual Coupling Reduction for UWB MIMO Antennas With a Wideband Neutralization Line,” Antennas Wirel. Propag. Lett. IEEE Antennas and Wireless Propagation Letters, vol. 15, pp. 166-169, 2016.

[5] S. Tripathi, A. Mohan, and S. Yadav, "A Compact Koch Fractal UWB MIMO Antenna With WLAN Band-Rejection,” Antennas Wirel. Propag. Lett. IEEE Antennas and Wireless Propagation Letters, vol. 14, pp. 1565-1568, 2015.

[6] N. K. Kiem, H. N. B. Phuong, Q. N. Hieu, and D. N. Chien, “A compact printed 4×4 MIMO-UWB antenna with WLAN band rejection,” 2013 IEEE Antennas and Propagation Society International Symposium (APSURSI), 2013.

[7] L. Liu, S. W. Cheung, and T. I. Yuk, "Compact MIMO Antenna for Portable UWB Applications With Band-Notched Characteristic,” IEEE Trans. Antennas Propagat. IEEE Transactions on Antennas and Propagation, vol. 63, no. 5, pp. 1917-1924, 2015.

[8] A. I. Najam, Y. Duroc, and S. Tedjni, “Uwb-Mimo Antenna With Novel Stub Structure,” PIER C Progress In Electromagnetics Research C, vol. 19, pp. 245-257, 2011.

[9] Y. Li, W. Li, C. Liu, and T. Jiang, "A printed diversity Cantor set fractal antenna for ultra wideband communication applications,” Isape2012, 2012..

[10] M. Jusoh, M. F. Jamlos, M. R. B. Kamarudin, and M. F. B. A. Malek, “A Mimo Antenna Design Challenges For Uwb Application,” Progress In Electromagnetics Research B PIER B, vol. 36, pp. 357-371, 2012. 